\title{
Inverse and Reciprocity Methods for Machinery Noise Source Characterization and Sound Path Quantification Part 2: Transmission Paths
}

\author{
Jan W. Verheij \\ TNO Institute of Applied Physics, P. O. BOx 155, 2600 AD DELFT, The Netherlands
}

In this article and in a foregoing companion article some novel approaches to the characterization of the noise source strength of machinery and to the ranking of transmission paths are reviewed. They form an addition to the more conventional approaches of the analysis of noise control problems in terms of source-transmission path-receiver schemes.

In the first article source strength descriptors have been defined in terms of equivalent fictitious elementary sources, such as acoustical monopoles and mechanical point forces. This second article presents examples, which illustrate how some of these unconventional source strength descriptors have been exploited for sound transmission path quantification. These concern applications in ships and road vehicles. In all cases it is the combination with experimental reciprocity techniques, that makes the use of the elementary substitution sources as source models very powerful for transmission path ranking. Therefore, an appendix on reciprocity relations in structural-acoustics is added to this article, including a brief bibliography on that subject.

\section{INTRODUCTION}

This article and the foregoing companion article ${ }^{1}$ are concerned with the experimental analysis of machinery noise control problems. Although the general approach, in terms of a source - transmission path - receiver scheme, is well-known, this is not the case with the manner in which source modelling is handled.

The noise control problems of interest here, are dealing with a multitude of simultaneous sources or with a single noise source like a machine, with a multitude of partial sources and 'parallel' transmission paths. The analysis models which are considered are limited to systems with supposedly linear behaviour, i.e. to systems for which output responses may be modelled as a linear superposition of all contributions of the partial sources and transmission paths. Then in loose mathematical terms, for example, the following model equation applies:

- m partial sources:

$$
O_{k}=\sum_{j=1}^{m} T F_{j, k} \times I_{j}
$$

This type of analysis requires a system modelling in terms of partial inputs $I_{j}$, which genuinely characterize the partial sources themselves and of output-input ratios $O_{k} / I_{j}$. These latter are transfer functions $T F_{j, k}$, which characterize the transmission paths.

In the first part of this article two examples are presented, which illustrate the use of the 'uncorrelated equivalent monopoles' method ${ }^{1}$ for the analysis of airborne sound trans- mission. The first example is concerned with the airborne sound transmission from a shipboard diesel generator set to underwater. The second example is concerned with the evaluation of the effectiveness of tightly fitting engine encapsulations in heavy road vehicles.

In the second part of this article, application of the 'equivalent correlated point forces' $\operatorname{method}^{1}$ for the analysis of structure-borne sound transmission will be illustrated for two examples of 'compact' sources. One is a hydraulic pump in a refuse vehicle and one is an electromechanical drive in a frame of a copier machine. A third application illustrates how this method of fictitious equivalent forces can be applied part way along a transmission path. This example concerns the sound transmission to underwater from the main gearbox in a ship via the flanking transmission path of a propeller shaft bearing.

\section{METHOD OF UNCORRELATED EQUIVALENT MONOPOLES}

\subsection{Experiments on a shipboard diesel engine}

In naval ships noisy machines are resiliently mounted to reduce the underwater sound radiation. However, the effectiveness of the vibration isolation may be spoiled to an unwanted degree by the 'flanking' airborne sound transmission. This might require costly extra noise reduction measures, like an enclosure. Therefore, the reliable analysis of the airborne sound transmission to the underwater in available ships is of great practical interest. The obvious approach of using loudspeakers as substitution sources is impractical in 